\title{
An Investigation on the Electrical Activity of the Brain During Fara- darmani Connection in the Fara-therapist Population
}

\author{
Mohammad Ali Taheri' ${ }^{1}$, Farid Semsarha ${ }^{2}$, Fateme Modarresi-Asem ${ }^{3}$ \\ ${ }^{1}$ Sciencefact Department, Cosmointel Ltd. Research Center, Ontario, Canada; taheri.m.a.2020@gmail.com \\ ${ }^{2}$ Institute of Biochemistry and Biophysics (IBB), University of Tehran, Tehran, Iran; semsarha@alumni.ut.ac.ir \\ ${ }^{3}$ Cancer Research Center, Shahid Beheshti University of Medical Sciences, Tehran, Iran; fm.asem@gmail.com \\ *Correspondence: Semsarha@alumni.ut.ac.ir; Tel.: +98-9121786577
}

\begin{abstract}
Mind-body interaction and its manifestations at the brain level has been studied extensively in the field of consciousness research. Fara-darmani Consciousness Field, as claimed by Mohammad Ali Taheri (the founder), is a method of connecting with the Cosmic Consciousness Network through human mind and his brain has a detective role in this process. As a result of this connection, the scanning process of the state of a being, e.g., the health status of the cells and consequently organs is performed. This study was conducted to evaluate the effects of the Faradarmani Consciousness Field connection on electroencephalogram (EEG) features as an important biomarker of the brain functioning. The results showed that there was a significant increase in the gamma2 frequency band $(35-40 \mathrm{~Hz})$ power in the frontal lobe in medial frontal gyrus (BA6) and paracentral lobule (BA31) of the brain during the task condition compared to the rest condition in a Fara-therapist population. Considering the cortical electrical activity of Fara-therapist's brain during Fara-darmani Consciousness Field connection, characterizing increase in the power of gamma wave and the activity of the areas affecting on memory, attention, perception and default mode network intrinsic activity. This manifestation distinguishes Fara-darmani Consciousness Field connection from other known methods dealing with the mind-body interaction criterion mainly different types of mediation.
\end{abstract}

Keywords: Consciousness Field; cosmic consciousness network; default mode network; EEG; Faradarmani; Fara-therapist; gamma wave; Mind-body

\section{Introduction}

Consciousness and what it is, is unknown to the world of science [1]. Where consciousness is placed, its internal and external dimensions, how we experience it and so on are important issues which are generally unanswered or with different answers based on the field of study. The role of the brain in providing the necessary conditions for conscious experience is interesting and challenging scientific topics today and is a common point in many related experimental studies [2].

One of the oldest methods of examining consciousness manifestations is by examining the electrical activity of the brain named electroencephalography (EEG). Hans Berger recorded the first human electroencephalograms (EEGs) in 1924 with the goal of discovering the physiological basis of psychic phenomena, however, the result of his study was led to characterizing some brain's wave patterns including $\alpha$ and $\beta$ waves and coining of the term "electroencephalogram" [3]. Later and with the help of numerous studies, the brain activity details based on cortical areas and waves frequencies were founded. The power of waves in the brain during different brain activities have been determined; the 
waves with higher than $30 \mathrm{~Hz}$ frequency (gamma waves) has been linked to diverse cognitive functioning, including general neural correlate contents of consciousness [4], conscious perception [5], solving binding problem [6], attention [7], working memory, and long term memory processes [8]. Other brain waves with lower than $30 \mathrm{~Hz}$ frequency, beta $(12-30 \mathrm{~Hz})$, alpha $(8-12 \mathrm{~Hz})$, theta (4$8 \mathrm{~Hz})$ and delta $(0.5-4 \mathrm{~Hz})$, have been presented as oscillating electrical voltages in different states of relaxed, very relaxed, deeply relaxed, and sleep, respectively [9]. Moreover, distinct cortical electrically active areas of brain (total of 52 Brodmann areas grouped into 11 histological areas) were determined [10]. These different parts of the cerebral cortex are involved in different cognitive and behavioral functions [11].

The distinction between task and rest states in the brain electrical activity measurements is the main step of consciousness related studies. Resting condition or basal state of the brain and its differences with the state of activity are described in several ways; Koch et al [12] in a brilliant review article described recent findings showing the anatomical neural correlates of consciousness are primarily localized to a posterior cortical hot zone that includes sensory areas rather than to a fronto-parietal network involved in task monitoring and reporting.

The default mode network (DMN) was originally described by Shulman et al. [13] and subsequently Raichle et al. (2001) [14], who observed that when a person is not focused on the outside world special brain regions experienced increased metabolic activity and decreased activity when engaged in a goal-directed (i.e., cognitively demanding) behavior. These regions also named default mode resting state network (DMRSN) constitute a set of brain areas: the ventral and dorsal medial prefrontal cortices (BA 24, 10m, 10r, 10p, 32a, 32c, 9), posterior cingulate/retrosplenial cortex (BA 29/30, 23/31), inferior parietal lobule (BA 39, 40), lateral temporal cortex (BA 21), and hippocampal formation [15]. Over the past two decades, it is founded that the default-mode network (DMN) has putative relationship to self-cognition [16] and mind wandering [17]. Moreover, it is founded that EEG gamma power over frontal and midline areas reflects default mode network (DMN) activity $[18,19]$.

Mind and body relationship investigation by applying task monitoring using various methods of meditation is one of the main area of consciousness studies in healthy persons and deals with task manifestations at brain level. Kuar and Singh [20] conducted a review of research into the effects of different types of meditation on brain waves. In this review, Buddhist meditation, Transcending meditation (TM), Yoga Meditation and some other lesser known types have been investigated and it turned out a rise in delta, theta, and alpha frequency bands in frontal, occipital and parietal brain areas identify different meditation states. Moreover, Desai et al [21] did a comprehensive review of fifteen authoritative articles on the various methods of yoga and their effects on the brain waves of individuals. In this study, it has been found that a variety of yoga techniques have a significant effect on increasing the alpha, beta, and theta waves in the frontal cortex. Lomas et al [22] in a systematic review of 56 papers, consisting of 1358 healthy individuals reveal that Mindfulness was associated with enhanced alpha and theta power (because of state of relaxed alertness). Moreover, no consistent patterns were observed in terms of beta, delta, and gamma in this study. Various studies emphasize that long-term meditation can suppress and reduce default mode processing and its related gamma wave power [18, 19, 23-25].

In addition to numerous studies on the various types of meditation with emphasize on the significant role of increasing alpha, delta, and theta waves and the reduction of gamma waves, few meditation practice found increase in high gamma wave (along with other meditation related waves and in regions without relation to brain default network) in the parietal and occipital regions in comparison with control, when meditators have a lot of experience (based on personal ability). Braboszcz et al [26] in three different meditation traditions (Vipassana, Himalayan Yoga and Isha Shoonya) showed higher parieto-occipital $60-110 \mathrm{~Hz}$ gamma (High gamma) amplitude in specific condition. Moreover, increased occipital gamma power related to long-term meditational expertise and enhanced sensory awareness studied [27] and some other previous related studies reviewed [28]. 
An innovative method in which each human mind (without any privileges in terms of experience or ability) is used as a medium to make a connection to special consciousness named whole or Cosmic Consciousness Network (CCN), is Fara-darmani Consciousness Field (CF) connection method. According to its founder, Mohammad Ali Taheri, Fara-darmani CF connection is completely different from meditation and other methods and techniques of working with mind in which experience has a special role in achievements and general position and the transcendence of consciousness is not considered.

In this approach, $\mathrm{CCN}$ is network of consciousness or the Internet of consciousness: the place that contains original and formative data and information of the universe and everything in it. Based on theoretical concepts of Fara-darmani, mankind through his mind is able to affect the overall health of himself and others in the world of matter and energy by connecting with the CCN [29]. The main principle of Taheri's approach is the "consciousness bond of the parts". Based on this concept each cell along with the molecules and their micro particles are made of vibrating fibers. Moreover, the factor that creates and directs these vibrations and movements is the consciousness that governs the universe $[29,30]$. For example, after the connection to $\mathrm{CCN}$, the scan of existential components, for example in humans, the health status of the cells and organs is automatically checked. Based on Faradarmani concepts, mankind has a higher status than any other living things in the cosmic ecosystem and his brain acts as a detector in the mentioned connection and any other cognitive processes of human life [29]. Since this ability must be manifested at the level of brain activity in humans, in this study we examined the electrical activity of the brains of people who have the ability to connect independently to the network of cosmic consciousness which named, Fara-therapist. 


\section{Materials and Methods}

This study is designed to test the effects of Fara-darmani CF on electroencephalography (EEG) features as an important biomarker of brain functioning. 36 healthy Fara-therapists (28 women and 8 men) in the age range of $20-50$ years $(M=36.5, S D=6.20)$ who have successfully completed the mentioned two-year training course, attended in the present study.

\subsection{Fara-darmani Consciousness Field application}

In order to apply the Fara-darmani $\mathrm{CF}$, in the Fara-therapist population, each person can announce the Fara-darmani CF for himself. In other words, a Fara-therapist states the connection between the subject under study (in this project himself) and the CCN, here named the announcement. Announcement is a process in which Taheri or any certified announcer declared and sent the important information of the subject under study to the Cosmic Consciousness Network by just recalling his/her name, the agreed time, and the location of the subject. The subject under study can be a patient or any individual who is willing to experience the Cosmic Consciousness Network. In the present study, subject of study is the announcer himself (Figure 1).

Fara-therapist (as announcer in Figure 1) according to Taheri is a trained and certified individual who took and completed specific training courses taught by Mohammad Ali Taheri or his certified masters. In these courses, which takes almost two years to complete under Taheri's supervision, the students learn theoretically and practically how to use the Consciousness Fields in Cosmic Consciousness Network.

Stablishing the connection to the CCN: a process that is available to everyone anywhere in the world, 24 hours a day. Apart from the present study, in which the announcers themselves have been the subject of the study, for any study/experience using the CFs, any researcher/volunteer must register on the COSMOintel Website (www.cosmointel.com). Once registered, go to the researcher/connection experience section and fill out a form. In order to study/experience at any given time and place, the researchers/volunteers simply need to introduce the testing center/himself to the guidance center. It should be noted that registration on the website and also requesting and gaining an announcement is free. 


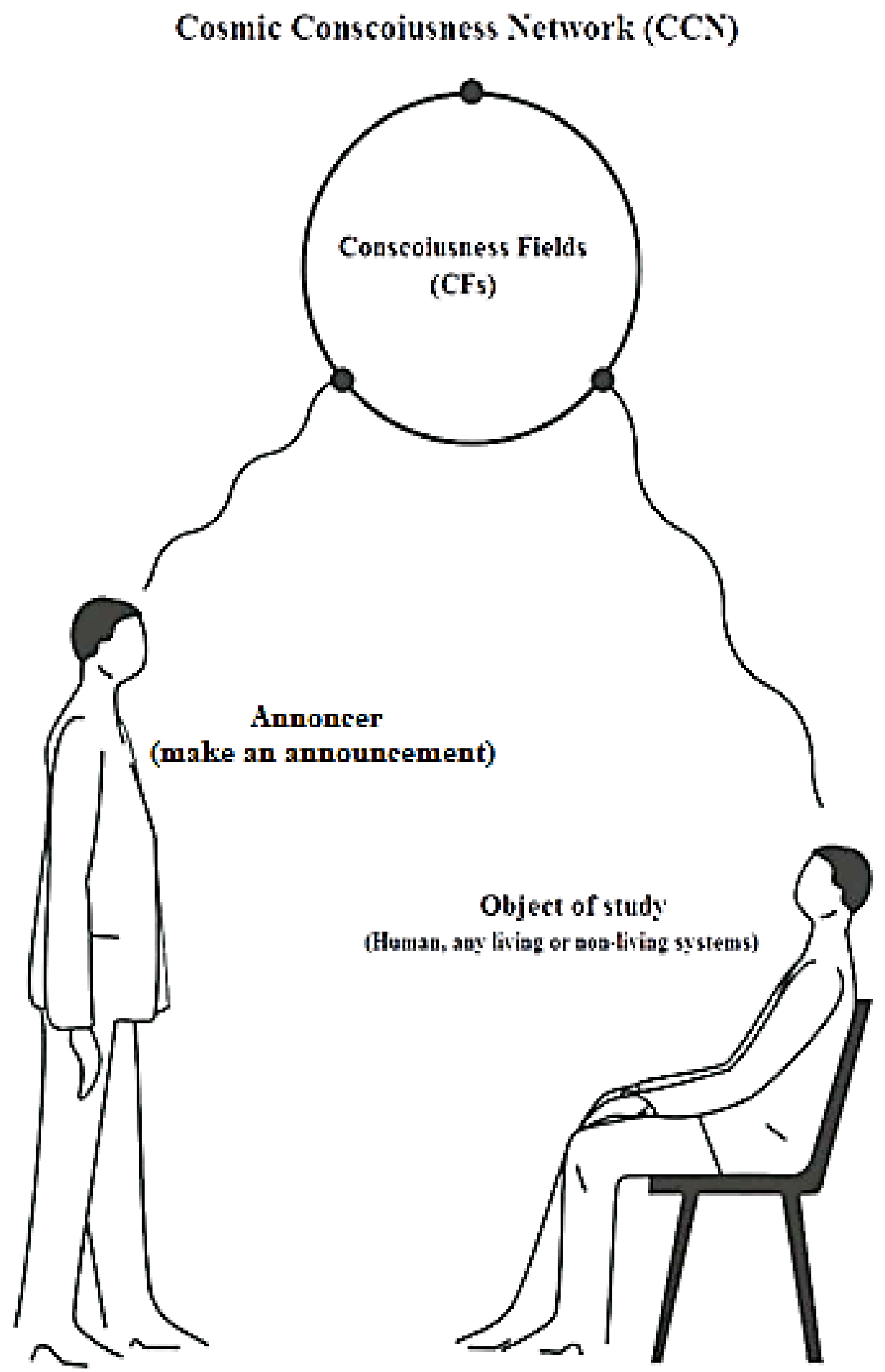

Figure 1 . How to announce and communicate between the subject of study and the Cosmic Consciousness Network (CCN) through $\mathrm{CF}$ according to Taheri. In the present study, announcer and object of study are the same. 


\subsection{EEG assay}

All volunteers were seated in a comfortably sound and light attenuated room, while 15 minutes or more of closed-eyes task/rest EEG data were collected by means of a 19-channel (Fp1, Fp2, F7, F3, Fz, F4, F8, T3, C3, Cz, C4, T4, T5, P3, Pz, P4, T6, O1 and O2) device (EEGR 19-26, Medicom company, Russia). In the task condition, the subject was asked to start Fara-darmani CF connection according to Taheri's approach.

Since different EEG frequencies reflect different functions, data were digitally filtered into ten frequency bands: delta $(1-4 \mathrm{~Hz})$, theta $(4-8 \mathrm{~Hz})$, alpha- $1(8-10 \mathrm{~Hz})$, alpha-2 $(10-12 \mathrm{~Hz})$, beta-1 $(12-15$ $\mathrm{Hz})$, beta-2 $(15-18 \mathrm{~Hz})$, beta-3 $(18-25 \mathrm{~Hz})$, high beta $(25-30 \mathrm{~Hz})$, gamma $1(30-35 \mathrm{~Hz})$ and gamma 2 $(35-40 \mathrm{~Hz})$. At least two minutes of artifact-free data were extracted from the EEG's total record for quantitative analysis. Power-spectral analysis was performed with Fast Fourier Transform (FFT). For each of the 19 monopolar derivations, absolute and relative power and mean frequency were computed for the mentioned frequency bands. Absolute power (uv Square) was the only parameter used to create the functional images of neuronal electrical activity for the 5 classical bands of EEG (delta, theta, alpha, beta and gamma).

\subsection{EEG-source localization analysis}

In this study the three dimensional intracerebral distribution of neuronal electrical activity or current density was assessed using Low Resolution Brain Electromagnetic Tomography (LORETA) with a resolution of $1 \mathrm{~Hz}$, from 1 to $40 \mathrm{~Hz}$. LORETA computes this parameter from the scalp-recorded potential distribution by assuming that the smoothest of all possible inverse solutions is the most plausible, which is consistent with the assumption of neighboring neurons are simultaneously and synchronously active [31]. The 3D solution space in LORETA was restricted to the cortical gray matter and hippocampus in the Talairach human brain atlas [32], as determined by the corresponding digitized Probability Atlas (Brain Imaging Center, Montreal Neurologic Institute). LORETA functional images of spectral density were estimated for the mentioned ten frequency bands (section 2.2).

\subsection{Statistical analysis}

The statistical method for comparing the individuals of each group in the two modes of rest and task was the t-test of dependent groups. The T-level thresholds were corresponding to statistically significant thresholds $(\mathrm{p}<0.05$ and $\mathrm{p}<0.01)$. 


\section{Results}

The results showed that there was a significant increase in gamma2 wave, $35-40 \mathrm{~Hz}$, on fronto-central areas of the brain in task condition compared the rest condition in this population (Figure 2).

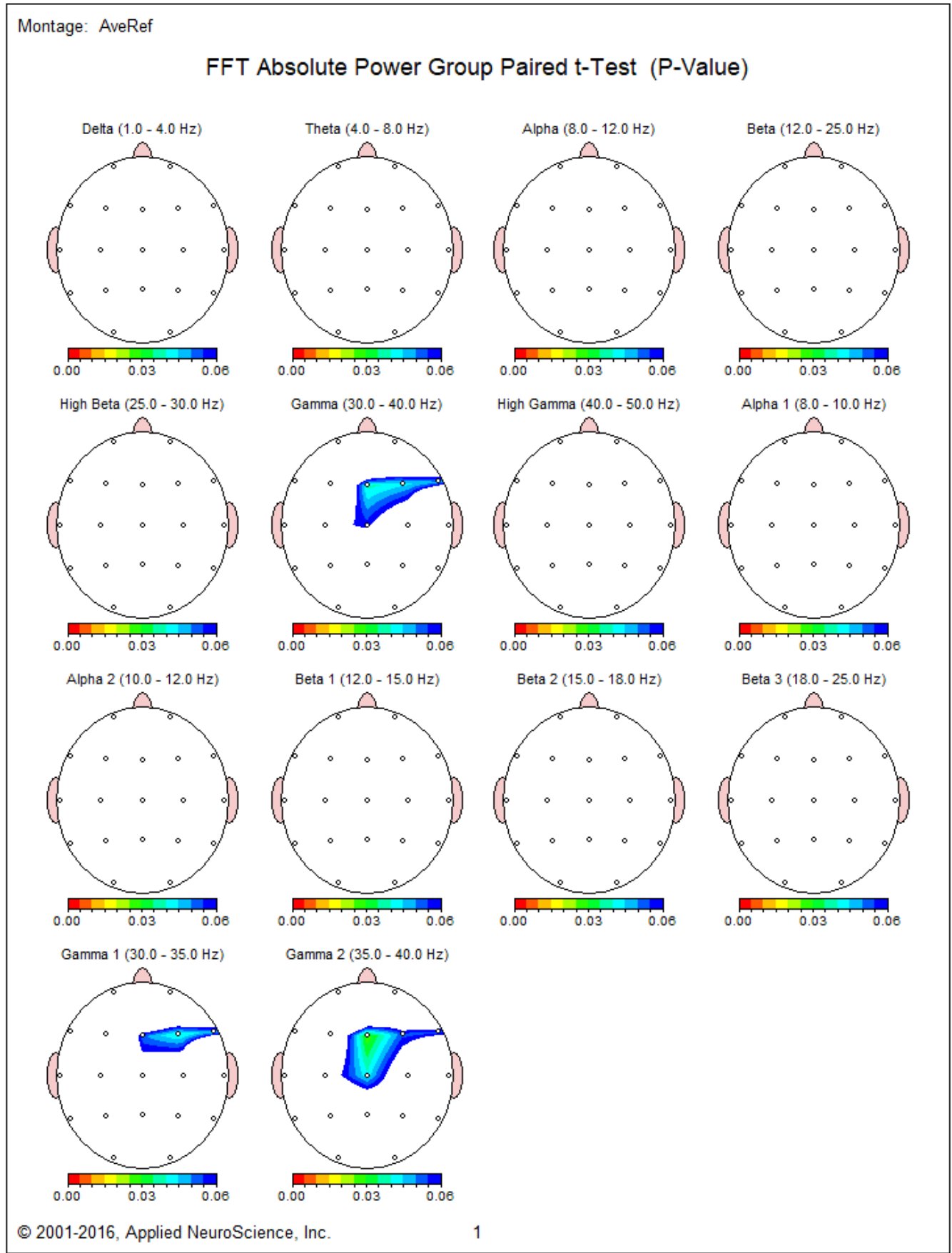

Figure 2: Gamma increase in tasks (Fz, F4, F8, Cz; Sig: $<0.05)$.

As shown in the table 1, the amount of increase in the power of gamma 2 wave, in $\mathrm{F} 4, \mathrm{~F} 8, \mathrm{Fz}$, and $\mathrm{Cz}$ channels are significant. The most significant increase of power is in the $\mathrm{Fz}$, and the most increase in comparison with the control is in the F8. 
Table 1: Power of gamma $2(35-40 \mathrm{~Hz})$ frequency band in all 19 channels in the rest and task and

\begin{tabular}{|c|c|c|c|c|c|c|c|}
\hline \multirow[b]{2}{*}{ Channel } & \multicolumn{2}{|l|}{ Rest } & \multicolumn{2}{|l|}{ Task } & \multicolumn{3}{|l|}{ Diff } \\
\hline & Mean/ $\mu \mathrm{V} 2$ & SD & Mean/ $\mu$ V2 & SD & Mean/ $\mu$ V2 & P value & Diff. Percent \\
\hline FP1-AVE & 0.79 & 0.47 & 0.97 & 0.72 & 0.19 & 0.22 & $23.80 \%$ \\
\hline FP2-AVE & 0.68 & 0.39 & 0.97 & 0.89 & 0.29 & 0.13 & $42.78 \%$ \\
\hline F3-AVE & 0.62 & 0.59 & 0.82 & 0.70 & 0.20 & 0.09 & $31.25 \%$ \\
\hline F4-AVE & 0.78 & 0.83 & 1.14 & 0.95 & 0.35 & 0.05 & $45.18 \%$ \\
\hline C3-AVE & 0.44 & 0.30 & 0.72 & 0.72 & 0.29 & 0.07 & $66.25 \%$ \\
\hline C4-AVE & 0.60 & 0.36 & 1.06 & 1.00 & 0.46 & 0.08 & $77.24 \%$ \\
\hline P3-AVE & 0.43 & 0.29 & 0.67 & 0.66 & 0.23 & 0.10 & $53.46 \%$ \\
\hline P4-AVE & 0.54 & 0.35 & 0.84 & 0.83 & 0.31 & 0.07 & $56.73 \%$ \\
\hline O1-AVE & 0.63 & 0.50 & 0.83 & 0.72 & 0.20 & 0.14 & $31.98 \%$ \\
\hline O2-AVE & 0.81 & 0.55 & 0.99 & 0.91 & 0.18 & 0.24 & $22.10 \%$ \\
\hline F7-AVE & 0.75 & 0.55 & 1.09 & 0.73 & 0.34 & 0.16 & $44.62 \%$ \\
\hline F8-AVE & 0.81 & 0.37 & 1.41 & 0.89 & 0.59 & 0.05 & $72.95 \%$ \\
\hline T3-AVE & 0.59 & 0.40 & 0.85 & 0.68 & 0.26 & 0.14 & $44.34 \%$ \\
\hline T4-AVE & 0.93 & 0.47 & 1.56 & 1.46 & 0.63 & 0.19 & $68.25 \%$ \\
\hline T5-AVE & 0.49 & 0.33 & 0.61 & 0.48 & 0.12 & 0.26 & $24.03 \%$ \\
\hline T6-AVE & 0.60 & 0.37 & 0.79 & 0.68 & 0.19 & 0.32 & $30.78 \%$ \\
\hline Fz-AVE & 0.62 & 0.57 & 0.89 & 0.79 & 0.27 & 0.03 & $43.82 \%$ \\
\hline Cz-AVE & 0.56 & 0.37 & 0.93 & 0.91 & 0.36 & 0.04 & $64.71 \%$ \\
\hline Pz-AVE & 0.48 & 0.32 & 0.75 & 0.75 & 0.26 & 0.09 & $54.74 \%$ \\
\hline
\end{tabular}


The LORETA results indicate a significant difference in the following areas. The high-precision point is the middle frontal. This area corresponds to the Broadman District 6. Moreover, second point is the paracentral lobule correspond to Broadman District 31. 2D and 3D representation of regions of activity in the brain of Fara-therapist in the task condition regarding the rest condition during Faradarmani consciousness filed announcement are shown in figures 3 and 4 , respectively.

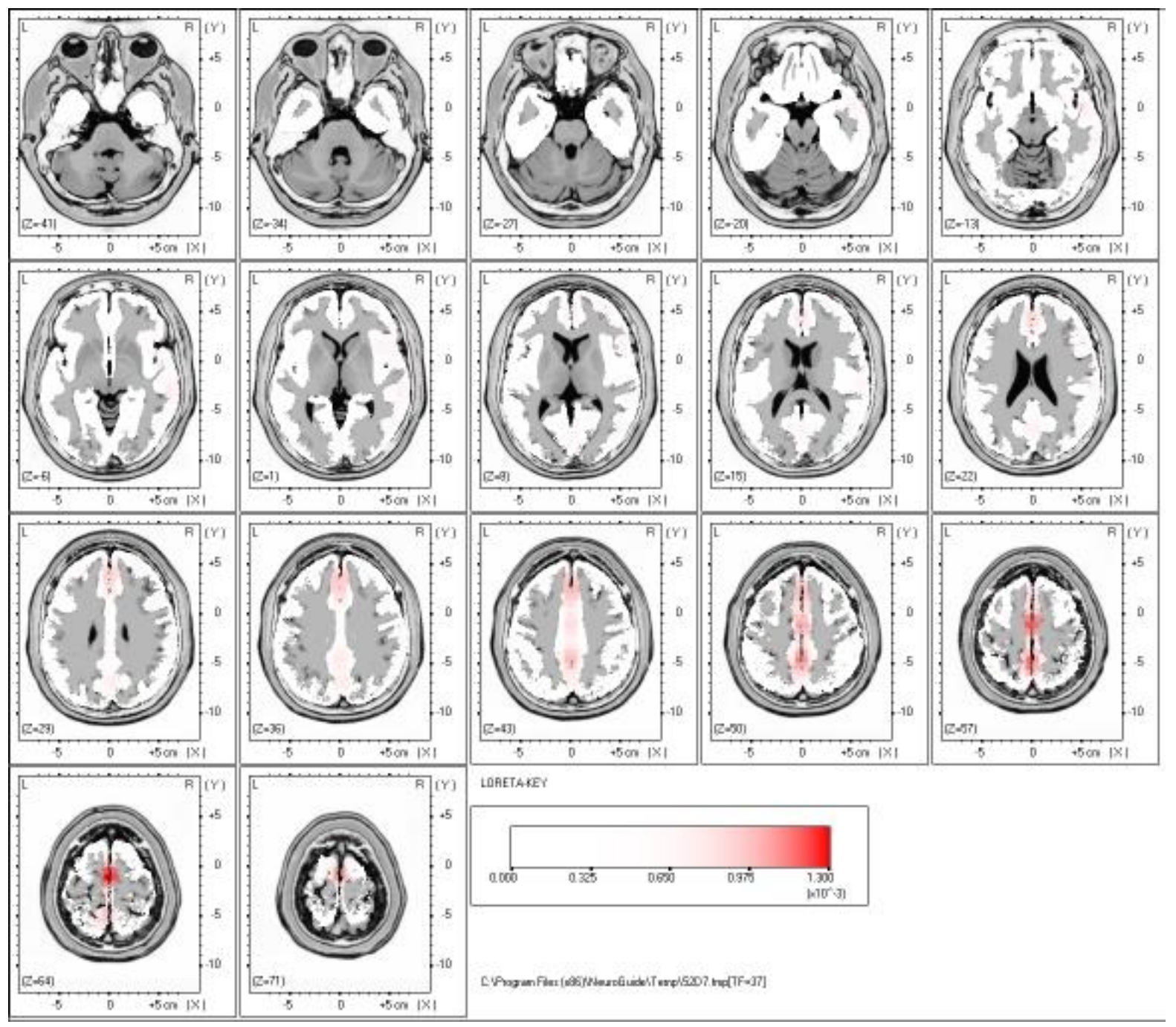

Figure 3: 2D regions of activity in brain of the Fara-therapist population during Fara-darmani connection. 


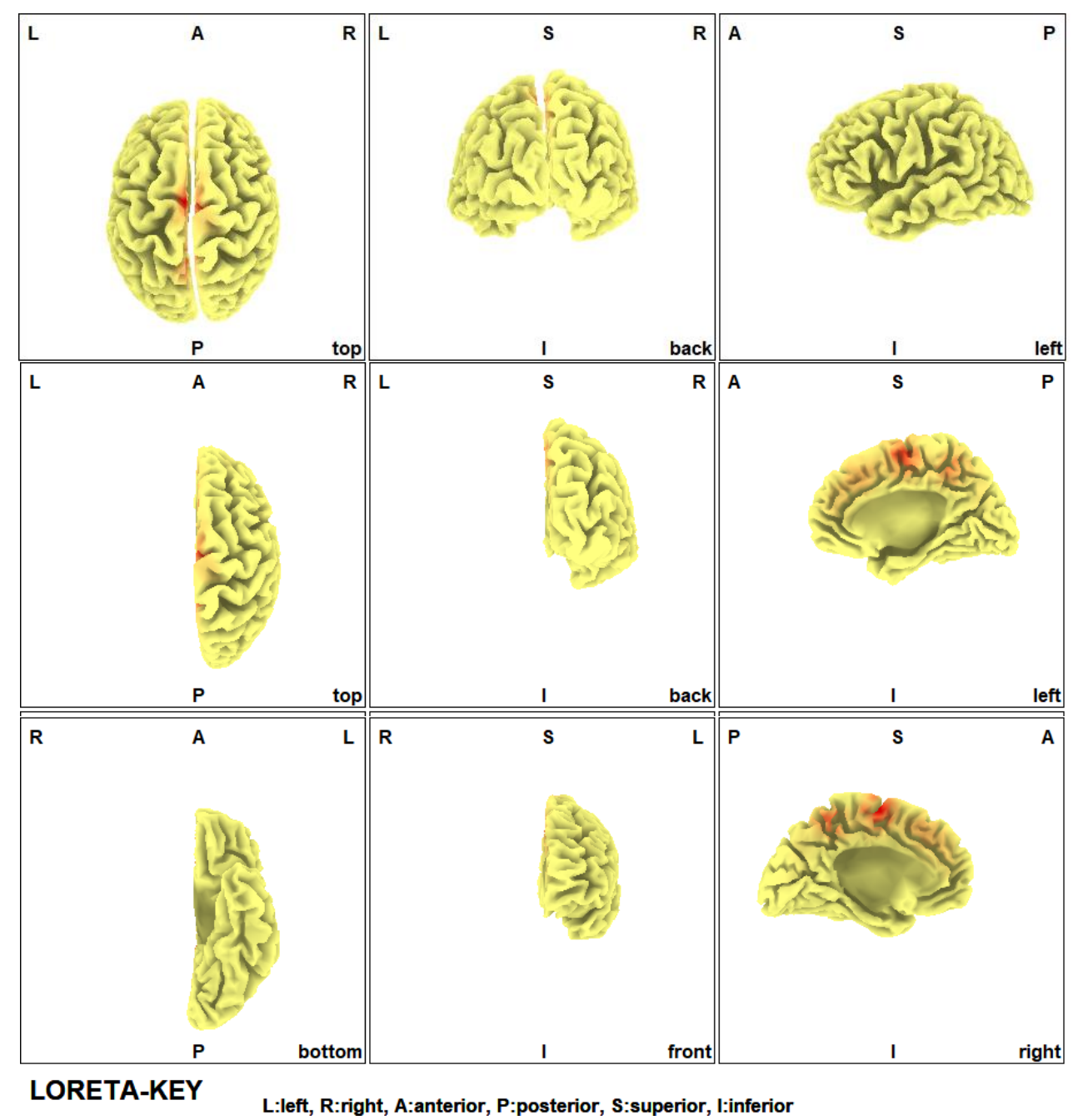

Figure 4: 3D regions of activity in brain of the Fara-therapist population during Fara-darmani connection.

\section{Discussion}

According to the founder, Fara-darmani is different and distinctive from other methods of mindbody interaction in the mechanism and purpose. In its mechanism, has been emphasized on the communication between the place of the whole consciousness (named by him Cosmic Consciousness Network: $\mathrm{CCN}$ ) containing the data and information of all the components of the universe and each living and inanimate components of the universe. Also, the aim of this connection is modifying and reconstructing the mentioned components. According to Taheri's approach, in the Fara-darmani connection, which is initiates by announcement of the Fara-therapists and by the operation of the $\mathrm{CCN}$, the human brain is like a detector and receiver that shows the manifestations of this connection and mediates its function.

In this study, which is similar to other methods of examining the mind-body interactions with the aim of investigating the electrical activity of the brain during Fara-darmani connection in the Faratherapist population, it observed that the brain activity during this connection is limited to the gamma waves in areas associated with the general and default mode network brain activity. A 
gamma wave is considered to be the fastest brain activity and responsible for cognitive functioning, learning, information processing, attention, focus, binding of senses (smell, sight, and hearing), consciousness, problem solving, mental processing, and perception.

On the other, two main activated brain areas of Fata-therapist population during Fara-darmani CF connection incorporated in multiple conscious experience different form most known mind-body interaction methods. First, BA6 area is associated with many functions mainly motor sequencing and planning movements. Participation of BA6 in memory, attention, and executive functions may be due to the activation of an extended brain network that sometimes involves this area [33]. Secondly, BA31 area, with main role in emotion is obvious as well as its participation in different types of memory (e.g., topographic memory, episodic memory, etc.) [34, 35]. Moreover, this area is one of the mentioned regions in the DMRSN (default mode resting state network) [15].

According to results of this study, although application of Fara-darmani is similar to various types of mind-body interaction in terms of mind mediation, relaxation and being in the present moment during the task, but the increase in gamma wave power in the frontal lobe (medial frontal gyrus (BA6) and paracentral lobule (BA31)), and the absence of low frequency waves increase during the Faradarmani CF connection indicate Fara-darmani connection manifestations are different than the other known methods of meditation and mind-body interaction. Moreover, according to the concepts of the method, based on establishing a connection and receiving the necessary structural and functional data and information of the organ and body from whole consciousness (CCN), the positive relationship of this connection and the increase in the power of gamma waves and activity of the effective parts of the brains in memory, attention, perception and motor movements is observed. Also in this case, during this connection, the increase in the intrinsic activity of the brain in relation to its general activities (DMN), which consumes more than $90 \%$ of the brain energy [36], is significant.

Further investigations on obtaining the graph of the electrical and metabolic activity of the brains during Fara-darmani connection in the Fara-therapist population in comparison with a non-Faratherapist population are the future related studies of the authors. Also, study on the samples of the patients with diseases related to the activated brain regions and increased wave frequencies seen in the present study will be of the special interest.

Author Contributions: "Conceptualization, methodology, supervision, Mohammad Ali Taheri, formal analysis, validation, project administration, Farid Semsarha. Investigation, writing-original draft preparation, Farid Semsarha, Fateme Modarresi-Asem.

Acknowledgments: The authors thank Mr. Behrouz Raadnasab for revising the English.

Conflicts of Interest: Mohammad Ali Taheri, as the founder of the main method of the present study and the guide on how to use it, is mentioned in the article, and all the executive steps of this study and data analysis have been done in double-blinded ways. 
References

1. Hameroff $S$ and R. Penrose. Consciousness in the universe: a review of the 'Orch OR' theory, Physics of Life Reviews 2014 11(1): 39-78.

2. Joseph E. LeDoux, Matthias Michel, and Hakwan Lauh. A little history goes a long way toward understanding why we study consciousness the way we do today. PNAS 2020 117(13): 6976-6984.

3. Millett D. Hans Berger: From psychic energy to the EEG. Perspectives in Biology and Medicine 200144 (4): 522-542.

4. Crick F. On consciousness. 1994 Nature 369: 86.

5. Meador K. J. Ray P. G, Echauz J. R. Loring D. W, Vachtsevanos G. J. Gamma coherence and conscious perception. Neurology 200259 (6): 847-854.

6. Buzsaki G (2006). "Cycle 9, The Gamma Buzz". Rhythms of the brain. Oxford. ISBN 978-0195301069.

7. Landau AN, Esterman M, Robertson LC, Bentin S, Prinzmetal W. Different Effects of Voluntary and Involuntary Attention on EEG Activity in the Gamma Band. Journal of Neuroscience 2007 27(44):1198611990. pmid:17978039.

8. Jensen O, Kaiser J, Lachaux J-P. Human gamma-frequency oscillations associated with attention and memory. Trends Neurosci. 2007 30(7):317-324.

9. Priyanka A. Abhang, Bharti W. Gawali, Suresh C. Mehrotra, Chapter 3 - Technical Aspects of Brain Rhythms and Speech Parameters. In Introduction to EEG- and Speech-Based Emotion Recognition, Editor(s): Priyanka A. Abhang, Bharti W. Gawali, Suresh C. Mehrotra, Academic Press, 2016, Pages 51-79,

10. Clarke E, Dewhurst K, Aminoff MJ. An Illustrated History of Brain Function: Imaging the Brain from Antiquity to the Present. Norman Publishing. 1996 ISBN 978-0-930405-65-6.

11. Tortora GJ, Derrickson BH. Principles of Anatomy and Physiology, 14th Edition, Wiley, 2013.

12. Koch C, Massimini M, Boly M, Tononi G. Neural correlates of consciousness: progress and problems. Nat Rev Neurosci. 2016 May;17(5):307-21. doi: 10.1038/nrn.2016.22. PMID: 27094080.

13. Shulman GL, Fiez JA, Corbetta M, Buckner RL, Miezin FM. Common blood flow changes across visual tasks: II. Decreases in cerebral cortex. J. Cogn. Neurosci. 1997 9: 648-63.

14. Raichle ME, MacLeod AM, Snyder AZ, Powers WJ, Gusnard DA, Shulman GL A default mode of brain function. PNAS 2001 98:676-82.

15. Buckner RL, Andrews-Hanna JR, Schacter DL. The brain's default network: anatomy, function, and relevance to disease. Ann N Y Acad Sci. 2008 Mar; 1124:1-38. doi: 10.1196/annals.1440.011. PMID: 18400922.

16. Gusnard D. A., Akbudak E., Shulman G. L., Raichle M. E. Medial prefrontal cortex and self-referential mental activity: relation to a default mode of brain function. Proc. Natl. Acad. Sci. U.S.A. 2001 98, 4259426410.1073/pnas.071043098

17. Mason MF, Norton MI, Van Horn JD, Wegner DM, Grafton ST, Macrae CN. Wandering minds: the default network and stimulus-independent thought. Science. 2007;315(5810):393-395. doi:10.1126/science.1131295

18. Berkovich-Ohana, A., Glicksohn J, and Goldstein A. Mindfulness-induced changes in gamma band activity - Implications for the default mode network, self-reference and attention. Clin. Neurophysiol. 2012 123:700710.

19. Berkovich-Ohana, A., J. Glicksohn, and A. Goldstein Studying the default mode and its mindfulnessinduced changes using EEG functional connectivity. Soc. Cogn. Affect. Neurosci. 2013 1-9.

20. Chamandeep Kaur, Preeti Singh, "EEG Derived Neuronal Dynamics during Meditation: Progress and Challenges", Advances in Preventive Medicine, vol. 2015, Article ID 614723, 10 pages, 2015. https://doi.org/10.1155/2015/614723.

21. Desai, R. et al. "Effects of yoga on brain waves and structural activation: A review." Complementary therapies in clinical practice 2015 (21) 2: 112-8.

22. Lomas T, Ivtzana I, Fua CHY. A systematic review of the neurophysiology of mindfulness on EEG oscillations. Neuroscience and Biobehavioral Reviews 2015 57: 401-410.

23. Brewer JA, Mallik S, Babuscio TA, Nich C, Johnson HE, Deleone CM, Minnix-Cotton CA, Byrne SA, Kober $\mathrm{H}$, Weinstein AJ, Carroll KM, Rounsaville BJ Mindfulness training for smoking cessation: results from a randomized controlled trial. Drug Alcohol Depend 2011119 (1-2): 72-80.

24. Garrison KA, Zeffiro TA, Scheinost D, Constable RT, and Brewer JA. Meditation leads to reduced default mode network activity beyond an active task. Cogn Affect Behav Neurosci. 2015 15(3): 712-720. doi:10.3758/s13415-015-0358-3. 
25. Judson A. Brewer, Patrick D. Worhunsky, Jeremy R. Gray, Yi-Yuan Tang, Jochen Weber, and Hedy Kober Meditation experience is associated with differences in default mode network activity and connectivity. PNAS 2011108 (50): 20254-20259.

26. Braboszcz C, Rael Cahn B, Levy J, Fernandez M, and Delorme A. Increased Gamma Brainwave Amplitude Compared to Control in Three Different Meditation Traditions. PLoS One. 2017; 12(1): e0170647.

27. Cahn BR, Delorme A, Polich J Occipital gamma activation during Vipassana meditation. Cogn Process 2010 11:39-56

28. Cahn, B., and J. Polich. Meditation states and traits: EEG, ERP, and neuroimaging studies. Psychol. Bull. 2006 132:180-211.

29. Taheri, M.A. “Human from another outlook” Interuniversal Press; 2nd Edition (September 26, 2013). ISBN13: 978-1939507006, ISBN-10: 1939507006

30. Taheri, M.A. The Purpose of Practicing Faradarmani Treatment; An Iranian Complementary and Alternative Medicine. Procedia - Social and Behavioral Sciences, 2014; 114: 75-79.

31. Pascual-Marqui RD, Michel CM, Lehmann D. Low resolution electromagnetic tomography: a new method for localizing electrical activity in the brain. Int J Psychophysiol 1994 18:49-65.

32. Talairach J, Tournoux P. Co-planar stereotaxic atlas of the human brain. 1988 Thieme, Stuttgart

33. Catalan M J, Honda M, Weeks R A, Cohen L G, Hallett M The functional neuroanatomy of simple and complex sequential finger movements: a PET study Brain 1998121 (Pt 2):253-64. doi: 10.1093/brain/121.2.253.

34. Berthoz A Parietal and hippocampal contribution to topokinetic and topographic memory Philos Trans $R$ Soc Lond B Biol Sci. 1997 Oct 29;352(1360):1437-48. doi: 10.1098/rstb.0130.

35. Krause B J, Horwitz B, Taylor J G, Schmidt D, Mottaghy F M, Herzog H, Halsband U, Müller-Gärtner H Network analysis in episodic encoding and retrieval of word-pair associates: a PET study. Eur J Neurosci 1999 11(9):3293-301. doi: 10.1046/j.1460-9568.1999.00723. x.

36. Raichlea ME and Snydera AZ A default mode of brain function: A brief history of an evolving idea. Neurolmage 2007 37: 1083-1090. 